\title{
Revisión
}

\section{Proteinuria, fisiología y fisiopatología aplicada}

\author{
Carlos Escalante-Gómez, Fernando Zeledón-Sánchez, Guido Ulate-Montero
}

\section{Resumen}

La proteinuria está definida por la presencia de proteínas en la orina. En los adultos se refiere a una excreción urinaria de estas superior a $150 \mathrm{mg}$ en 24 horas. Se ha utilizado como un marcador de lesión renal, constituyéndose en uno de los datos más importantes para el nefrólogo. Sin embargo, patologías tan comunes como la hipertensión arterial y la Diabetes Mellitus frecuentemente manifiestan sus afecciones renales con la presencia de proteinuria, convirtiéndose ahora en un marcador de enfermedades sistémicas y no solo renales. Normalmente, un individuo filtra $5000 \mathrm{mg}$ de proteínas cada día, de los cuales $4950 \mathrm{mg}$ son reabsorbidos en el túbulo proximal del riñón, de manera que la cantidad excretada es poca. En el presente artículo se exponen los diferentes tipos de proteinuria con base en conceptos fisiopatológicos. Hay varios métodos de laboratorio que permiten la cuantificación de la proteinuria, siendo la relación proteinuria / creatinuria y la orina de 24 horas las más utilizadas. La relevancia de esta revisión se muestra al tomar en cuenta que la proteinuria es el factor aislado más importante para determinar el avance y progresión de la enfermedad renal. También se ha mostrado que el riesgo añadido por la presencia de proteinuria fue superior al causado por el tabaco, la diabetes o la hipertrofia ventricular izquierda para la presencia de eventos isquémicos cardiovasculares. La proteinuria es más que solo proteínas en la orina, es una señal de alerta.

Descriptores: proteinuria, fisiología renal, nefropatía

Key Words: proteinuria, renal physiology, nephropathy

Recibido: 17 de mayo de 2006

Aceptado: 2 de noviembre de 2006

Departamento de Fisiología, Universidad de Costa Rica. Programa de Maestría en Fisiología de Sistemas.

Abreviaturas:

AA, aminoácidos; IC, intervalo de confianza; $\mathrm{kDa}$, kilodalton; nm, nanómetro

Correspondencia: Carlos Escalante Gómez Apto. 783-2400, San José, Costa Rica dr.escalante@gmail.com

ISSN 0001-6002/2007/49/2/83-89 Acta Médica Costarricense, (C2007 Colegio de Médicos y Cirujanos
Históricamente, la proteinuria se ha utilizado como un marcador de lesión renal, constituyéndose en uno de los datos más importantes para el nefrólogo. Sin embargo, con los avances en los campos de la fisiología, la fisiopatología y la medicina interna, el estudio y manejo de algunas proteinurias ha pasado de las manos de los especialistas hacia las de los médicos de atención primaria. Patologías tan comunes como la hipertensión arterial y Diabetes Mellitus frecuentemente manifiestan sus afecciones renales con la presencia de proteinuria, convirtiéndose ahora en un marcador de enfermedades sistémicas y no solo renales.

No todas las proteinurias son iguales. Existen proteinurias "aisladas", las cuales no se asocian con disfunción renal patológica, como lo es la proteinuria ortostática, que solo requieren más que un seguimiento periódico. Pero también existen proteinurias "asociadas", producto de alguna disfunción renal o sistémica, como lo es un síndrome nefrótico, que requieren la participación activa del médico para evitar el progreso a una falla renal severa. Es únicamente con el conocimiento del funcionamiento renal normal y la manera como las enfermedades pueden alterar este equilibro, que se logra entender los mecanismos por los cuales se produce la proteinuria. En esta revisión se abarca la definición, fisiología, fisiopatología, métodos de diagnóstico y algunas generalidades del tratamiento de las proteinurias, con el propósito de que el médico tenga un concepto integral de lo que significa la presencia de proteínas en la orina. 


\section{Definición}

La proteinuria es la presencia de proteínas en la orina. Clínicamente, en adultos, una excreción urinaria de proteínas superior a $150 \mathrm{mg}$ en 24 horas, define la proteinuria. ${ }^{1} \mathrm{En}$ niños este criterio varía según la edad y el peso. En neonatos (<30 días) es de $145 \mathrm{mg} / \mathrm{m}^{2} / 24 \mathrm{hrs}$, en lactantes ( 1 año), 110 $\mathrm{mg} / \mathrm{m}^{2} / 24 \mathrm{hrs}$ y en niños (2 a 10 años), $85 \mathrm{mg} / \mathrm{m}^{2} / 24 \mathrm{hrs}{ }^{2,3}$

\section{Proteínas plasmáticas - albúmina}

Frente a las 3000-5000 proteínas intracelulares estudiadas, solo se han descrito algunos cientos de proteínas plasmáticas. Con excepción de las hormonas peptídicas y de las inmunoglobulinas, las proteínas circulantes son de síntesis preferentemente hepática, ${ }^{4}$ algunas de ellas son los factores de coagulación, los factores del sistema de complemento, las lipoproteínas y las proteínas reactivas de fase aguda. La concentración normal de las proteínas en suero es de 6.6 a $8.7 \mathrm{~g} / \mathrm{dl} .{ }^{4,5}$

La principal proteína plasmática es la albúmina, la cual tiene una concentración de $4.0 \mathrm{~g} / \mathrm{dL}$ en suero (representa cerca del el $50-60 \%$ de las proteínas plasmáticas). Tiene un peso de $66 \mathrm{kD}$, está compuesta por $585 \mathrm{AA}$ y es de forma elíptica. Se producen de 9 a $12 \mathrm{~g}$ c/d y es muy soluble. Es predominantemente extravascular, con un total de $160 \mathrm{~g}$ en el intersticio y $140 \mathrm{~g}$ en el volumen circulatorio. Alrededor del $5 \%$ se filtra por los capilares cada hora, y se recicla cada 18 horas, por medio del sistema linfático. La albúmina aporta el $70 \%$ de la presión oncótica intravascular. ${ }^{5}$ Dentro de las funciones más importantes de la albúmina están: ser una molécula de transporte (carga negativa débil), comportarse como una molécula antioxidante (grupo tiol), modular la filtración capilar (presión oncótica), modular la coagulación como antitrombótico (prolonga la vida media del óxido nítrico) y como búffer. ${ }^{6,7}$

\section{Manejo renal de proteínas}

En cuanto al manejo renal de las proteínas, se observará que cada parte del riñón desempeña un papel determinante (Figura 1).

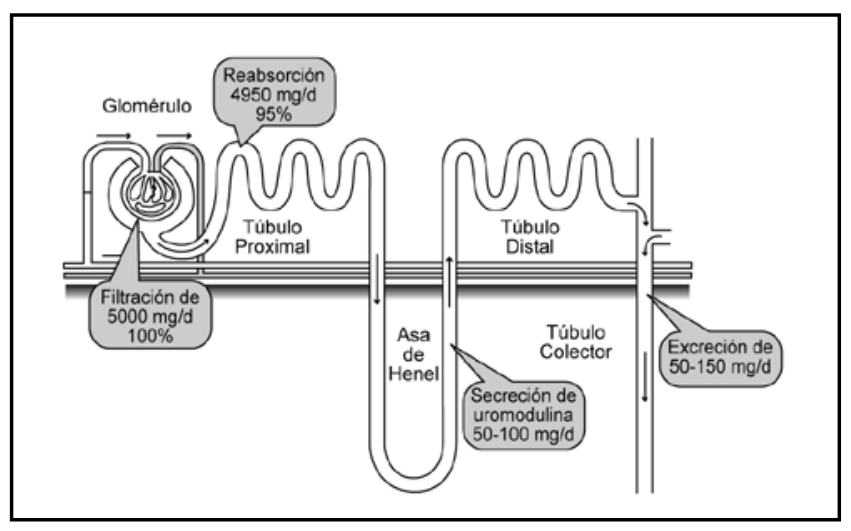

Figura 1. Manejo renal de las proteínas
En un individuo adulto, la masa filtrada diariamente, es decir, el producto de la tasa de filtración glomerular por la concentración de proteínas en el filtrado, es $5 \mathrm{~g} / \mathrm{d} .^{5} \mathrm{La}$ filtración proteica está determinada por las diferentes capas de la membrana filtrante glomerular. La primera capa, el endotelio vascular, que presenta fenestraciones de hasta 70 $\mathrm{nm}$, limita el paso de los componentes celulares del plasma $\mathrm{y}$, generalmente, no tiene un papel limitante respecto al agua y a los solutos pequeños. ${ }^{5,8}$ Luego la membrana basal glomerular, que tiene un grosor de aproximadamente 300$350 \mathrm{~nm}$, está compuesta por tres capas: la lámina rara interna, la lámina densa y la lámina rara externa. Los componentes principales de esta capa son el colágeno tipo IV, la laminina, el nidógeno y los proteoglicanos (perlecan y agrina), ${ }^{9} \mathrm{de}$ manera que constituyen un filtro físico para moléculas con un peso molecular superior a $1 \mathrm{kDa}$, y además tiene carga negativa, que complementa su función de filtración. ${ }^{5,10} \mathrm{~A}$ pesar de que antes se consideraba esta segunda capa como la principal determinante de la filtración glomerular, se ha visto que es la tercera capa la que define las características del ultrafiltrado. La tercera capa está compuesta por elementos celulares llamados pedículos, los cuales están unidos entre ellos por "slit diaphragms", que son los principales limitantes de la filtración, contienen poros de 4 a $14 \mathrm{~nm}$ y presentan glicoproteínas con cargas negativas. Ante el microscopío electrónico da la apariencia de una matriz molecular compleja. La nefrina es el principal componente proteico determinante de la filtración a este nivel, pero además hay otras proteínas que participan, como la podocina, la CD2AP, y la neph-1. ${ }^{10,11}$ Aunque ha habido grandes avances en estos estudios, todavía observa la filtración de algunas sustancias, como la albúmina, que en teoría, no deberían filtrarse. Basándose en esto, se han desarrollado varias teorías de modelos matemáticos que explicarían la filtración de tales sustancias. La más aceptada es la que propone la existencia de un isoporo (4-5 $\mathrm{nm}$ ) que limita la filtración "normal", pero además refiere la existencia de "shunts" no selectivos $(14 \mathrm{~nm})$, los cuales permitirían el paso de algunas sustancias grandes o muy negativas. ${ }^{12}$

La reabsorción tubular renal desempeña un papel muy importante para evitar la depleción proteica corporal. Diariamente, la masa reabsorbida (masa filtrada - masa excretada) es cercana a $4950 \mathrm{mg} / \mathrm{d}$. La reabsorción proteica ocurre principalmente en el túbulo proximal, en las porciones S1 y S2. ${ }^{5,13}$ Es una reabsorción mediada por endocitosis, la cual se produce gracias a la participación de las proteínas megalina y cubilina, que actúan como receptores en el lumen tubular. ${ }^{9}{ }^{13}$ La megalina tiene un peso molecular de $600 \mathrm{kDa}$ y pertenece a la familia de LDLR. En su estructura presenta 4 agrupamientos de cisteína, los cuales funcionan como puntos de unión para sus diferentes ligandos, principalmente para las proteínas y el calcio. ${ }^{14}$ La cubilina tiene un peso molecular de $460 \mathrm{kDa}$ y también se conoce como el "receptor del factor intrínseco del intestino". Presenta 27 dominios CUB, los cuales participan en la unión de sustancias. De las dos proteínas, la megalina es la principal (más de 40 
ligandos), mientras que la cubilina (aprox. 15 ligandos) llega a complementar la función de la primera. ${ }^{13,14}$

La secreción proteica tubular tiene lugar solo en la parte ascendente del asa de Henle. ${ }^{5,8}$ La única proteína descrita hasta ahora, secretada hacia el lumen tubular, es la proteína de Tamm-Horsfall, también conocida como uromodulina. Se trata de una glicoproteína del tipo fostatidilinositol, de 95 $\mathrm{kDa}$ de peso molecular y consta de $616 \mathrm{AA}$; es formada exclusivamente en el riñón y es secretada en al asa ascendente de Henle. UMOD es el gene encargado de codificar la uromodulina y se encuentra en el cromosoma 16 p. ${ }^{15,16}$ Es la proteína urinaria más común en condiciones fisiológicas y se pueden secretar desde 50 hasta $200 \mathrm{mg} / \mathrm{d}$ (masa secretada). Tiene 48 residuos de cisteína que le dan una conformación única y expone 8 sitios para glicosilación y enlace. ${ }^{16}$ También contiene un dominio que funciona como receptor para el calcio. Dentro de sus funciones se han descrito: 1-formar agregados linfocitarios e interferir con la función linfocitaria: puede modular la función de citokinas a nivel tubular; 2competir con la uroplakina para la adhesión de fimbrias de Escherichia coli tipo 1, proveyendo protección contra las infecciones, lo que logra al unirse a la adhesina FimH; 3inhibir la agregación de cristales de oxalato de calcio en el lumen tubular. ${ }^{17-19}$

\section{Métodos de laboratorio para determinar la proteinuria}

Hay diferentes opciones para determinar o cuantificar la presencia de proteínas en la orina. El "dipstick" tiene la ventaja de ser un método rápido y barato, sin embargo, ofrece muchos falsos positivos. Se basa en un método colorimétrico y da los resultados en rangos: negativo (0-10 $\mathrm{mg} / \mathrm{dL})$, trazas $(10-20 \mathrm{mg} / \mathrm{dL}),+(30 \mathrm{mg} / \mathrm{dL}),++(100 \mathrm{mg} /$ $\mathrm{dL}),+++(300 \mathrm{mg} / \mathrm{dL}) \mathrm{y}++++(1000 \mathrm{mg} / \mathrm{dL})$. Es bastante sensible para la albúmina, pero no detecta proteínas pequeñas como las macro y micro globulinas ni las proteínas Bence Jones. ${ }^{3,20,21}$

Existe otro método, que cada vez se usa menos, y se basa en el ácido sulfosalicílico (SSA). Es una prueba cualitativa basada en la turbidez comparada, es más sensible para proteínas de bajo peso y logra detectar niveles desde 4 $\mathrm{mg} / \mathrm{dl}^{21}$

En los últimos 5 -7 años se ha comenzado a utilizar más la relación proteinuria / creatinuria, con el fin de cuantificar la cantidad de proteinuria en una muestra aislada. Se ha observado una buena correlación con la orina de 24 hrs. y es fácil de interpretar: por ej. una relación de $0.2=0.2$ grs proteínas / $24 \mathrm{hrs}$; relación $3.5=3.5$ grs / $24 \mathrm{hrs}$. No se debe utilizar la primera muestra matutina para esta relación proteinuria/creatinuria, dado que los cambios en la tasa de filtración renal, por estar acostado mucho tiempo, podría dar un resultado mayor de lo real..$^{22-24}$

La orina de 24 hrs sigue siendo la prueba de oro para el estudio de la proteinuria. Se descarta la primera orina del día y se continúa la recolecta hasta la del día siguiente. La ventaja de la orina de 24 hrs no es solo la posibilidad de cuantificar la proteinuria total, sino que también permite identificar cuáles proteínas se están secretando para poder clasificar las proteinurias y orientarse en el posible mecanismo fisiopatológico. ${ }^{25-27}$

\section{Clasificación fisiopatológica}

A lo largo de los años han surgido diversas clasificaciones de la proteinuria, sin embargo, algunas, más que simplificar el entendimiento de la patología, la han complicado. En la presente revisión se expondrá una clasificación que pretende simplificar el panorama. La proteinuria se puede dividir en dos grandes grupos (cuadro 1): la proteinuria aislada (no asociado a patología renal o sistémica) y la proteinuria asociada (evidencia de patología renal o sistémica).

\section{Proteinuria aislada}

\section{Proteinuria funcional}

Es una forma común de proteinuria que se presenta asociada a la fiebre, ${ }^{28}$ el ejercicio excesivo, ${ }^{29,30}$ la exposición al frío, el estrés emocional, las convulsiones e inclusive al embarazo normal. ${ }^{31,32}$ A este tipo de proteinuria se le conoce como funcional porque no existen defectos renales intrínsicos estructurales ni funcionales. Es causada por cambios hemodinámicos que favorecen un aumento en la filtración glomerular de las proteínas plasmáticas. Se suele resolver espontáneamente unos días después de la noxa precipitante y no se asocia con patología renal progresiva. En el caso de la proteinuria postejercicio, se han identificado tantos aumentos en la permeabilidad glomerular como descensos en la reabsorción tubular.

\section{Proteinuria transitoria idiopática}

Este tipo de proteinuria es bastante frecuente en niños, adolescentes y adultos jóvenes. Representa la variedad más común de las proteinurias benignas. ${ }^{33}$ Estos pacientes son asintomáticos y generalmente se descubre la proteinuria en algún examen de control o tamizaje. No existe sedimento urinario característico y suele resolverse espontáneamente, de manera que no aparece en otros controles. No se han

\section{Cuadro 1.Clasificación de la proteinuria}

- Proteinuria aislada benigna

1. Proteinuria funcional

2. Proteinuria transitoria idiopática

3. Proteinuria intermitente idiopática

4. Proteinuria ortostática / (postural)

- Proteinuria aislada persistente

- Proteinuria asociada

1. Proteinuria no nefrótica

2. Proteinuria nefrótica 
documentado riesgos en el largo plazo asociados con este tipo de proteinuria, y probablemente se debe a cambios transitorios en el perfil hemodinámico renal. ${ }^{34} \mathrm{El}$ paciente no amerita biopsia renal y su control consiste en repetir las muestras dos o tres veces más, para determinar si en efecto la proteinuria es transitoria, intermitente o persistente; esto debe hacerse antes de abarcar estudios complementarios innecesarios.

\section{Proteinuria intermitente idiopática}

Los pacientes con este tipo de proteinuria benigna presentan proteinuria independiente de la posición corporal en al menos un $50 \%$ de las muestras de orina recogidas al azar, pero no en todas. ${ }^{35-37}$ La mayoría de los pacientes son jóvenes, menores de 30 años, presentan exámenes de función renal normal y mantienen presiones arteriales normales. En biopsias renales, se ha mostrado que hasta el $60 \%$ de los pacientes pueden presentan lesiones glomerulares leves que incluyen esclerosis e hipercelularidad. Sin embargo, estudios de seguimiento de hasta 40 años, han indicado que la evolución de estos pacientes, en ausencia de enfermedades sistémicas asociadas, es bastante buena y no difieren de la de la población general. En este grupo, la progresión a la insuficiencia renal es poco probable, de manera que se recomienda un seguimiento anual, pero generalmente no amerita manejo especializado.

\section{Proteinuria ortostática (postural)}

Es probablemente la variedad de proteinuria benigna mejor estudiada. ${ }^{38-44} \mathrm{La}$ excreción proteica es mayor, independiente de la condición, al estar de pie que al estar acostado. ${ }^{41}$ Sin embargo, las personas con proteinuria ortostática no presentan proteinuria cuando están acostadas; este punto es crítico en el diagnóstico, dado que los pacientes nefrópatas sí presentan proteinuria al estar en esa posición. La mayoría de las personas con proteinuria ortostática tienen excreciones diarias menores a $2 \mathrm{~g}$ /día; estos pacientes no pueden ser portadores de hipertensión arterial y deben tener pruebas de función renal normal. Afecta principalmente a los adolescentes y rara vez se diagnostica en pacientes mayores de 30 años. ${ }^{38,39}$ Existen dos variedades 1-transitoria, la cual solo se evidencia en unas cuantas oportunidades, y probablemente corresponde a una proteinuria funcional; 2persistente, la cual se presenta a lo largo de los años y generalmente mejora y desaparece con el crecimiento, durante la adolesencia. El mecanismo fisiopatológico aún no se ha determinado, sin embargo, se ha mostrado que debe existir algún factor renal predisponente asociado con alguna alteración hemodinámica renal. ${ }^{43}$ Los estudios que han incluido biopsias renales han mostrado, en ocasiones, cambios renales mínimos similares a la hipercelularidad mesangial y expansión mesangial. ${ }^{44}$ Recientemente se han mostrado depósitos mesangiales de complementos (C3 y C4) en casi todas las biopsias relacionadas con proteinuria ortostática. ${ }^{45}$ Los cambios hemodinámicos asociados a este tipo de proteinuria corresponden a dos modelos: 1) los pacientes que presentan una hipersensibilidad hacia la vasoconstricción arteriolar mediada por la angiotensina II, y 2) una compresión física de alguna de las venas renales (principalmente la izquierda) ${ }^{46}$, de manera que se pueda producir a una proteinuria por sobrecarga en un riñón ya predispuesto (depósitos de $\mathrm{C} 3 / \mathrm{C} 4) .{ }^{43}$ En estudios en el largo plazo (20 años) se ha mostrado que el pronóstico es bastante bueno. ${ }^{39}$ Solamente un $15 \%$ de los pacientes continuarán presentando proteinuria luego de los 30 años y rara vez alguno avanzará hacia la insuficiencia renal.

\section{Proteinuria aislada persistente}

Corresponde a un grupo de pacientes con proteinuria en todas o casi todas las muestras tomadas al azar, sin importar su posición (de pie / acostado), que no presentan patología asociada o alteraciones en las pruebas de función renal. ${ }^{47,48}$ Se manifiesta en niños, adolescentes y adultos jóvenes, y existe una prevalencia masculina. Algunos estudios han mostrado que hasta un $60 \%$ tienen una alteración renal histológica de tipo mínimo, que algunos autores proponen como cambios iniciales de lesiones glomerulares. ${ }^{49-51}$ Actualmente no existen estudios en el largo plazo que abarquen esta población. Al comprender un grupo muy heterogéneo que puede presentar grandes variaciones en la proteinuria y cambios histológicos, la gran mayoría de los expertos recomienda un seguimiento periódico.

\section{Proteinuria asociada}

\section{Proteinuria no nefrótica}

La proteinuria no nefrótica se refiere a las proteinuria en el rango de 150 a $3,500 \mathrm{mg} / 24 \mathrm{hrs}$. Una proteinuria asociada con valores menores a $2 \mathrm{~g} / 24 \mathrm{hr}$ no excluye patología glomerular, sin embargo, se deben considerar patologías tubulointersticiales y vasculares como la nefritis intersticial, la nefropatía por reflujo, el riñón poliquístico, la nefropatía medicamentosa (AINES/aminoglucósidos), exposición a metales pesados y las nefropatías asociadas a hipercalemia e hiperuriciemia. También debe recordarse que la necrosis tubular aguda puede llevar a proteinuria de hasta $2 \mathrm{gr} / 24 \mathrm{hr}$. Algunas enfermedades sistémicas como por ejemplo, la hipertensión arterial, el lupus eritematoso sistémico y las discrasias sanguíneas, pueden producir estas proteinurias. ${ }^{10,20,27}$

La microalbuminuria, una variedad de proteinuria glomerular, se define como la excreción de 30 a 300 mg de albúmina en $24 \mathrm{hrs}$. La microalbuminuria generalmente se detecta temprano en los pacientes diabéticos y se puede usar como un marcador pronóstico de la nefropatía diabética en pacientes insulina-dependientes. ${ }^{21,52}$

La proteinuria tubular rara vez excede $2 \mathrm{gr} / 24 \mathrm{hr}$. Es el resultado de una reabsorción inadecuada del filtrado proteico normal del glomérulo. Este tipo de proteinuria se caracteriza por presentar proteínas de baja masa molecular, principalmente las beta ${ }_{2}$-microglobulinas y otras como las 
lisozimas e insulina. Estas pequeñas proteínas se filtran normalmente por el glomérulo y son reabsorbidas $y$ degradadas en el túbulo proximal; en presencia de lesión tubular estas proteínas serán excretadas en su forma original. En la fisiopatología de la proteinuria tubular la albúmina tiene un papel secundario. ${ }^{20,21,27}$

La proteinuria por sobrecarga se presenta cuando hay un aumento importante en la concentración plasmática de proteínas, de manera que aumenta la filtración proteica en los glomérulos normales. Esta carga de proteínas, sobrepasa la capacidad tubular de reabsorción y, por ende, aumenta la excreción. El mieloma múltiple, al presentar una gran cantidad de cadenas livianas monoclonales, es una causa relativamente común de proteinuria por sobrecarga; otras incluyen: la hemoglobinuria por anemia hemolítica, la mioglobinuria en la rabdomiolisis severa y la lisosimuria en la leucemia mielomoncítica. ${ }^{53}$

\section{Proteinuria nefrótica}

El síndrome nefrótico, como tal, incluye una tétrada diagnóstica: proteinuria mayor a $3.5 \mathrm{grs} / 1.73 \mathrm{~m}^{2} / 24 \mathrm{hrs}$, hipoalbuminemia, edema e hiperlipidemia. ${ }^{27,53}$ La gran pérdida urinaria de proteínas lleva a la hipoalbuminemia y esta a los edemas e hiperlipidemia, lo cual predispone al paciente a eventos tromboembólicos, disfunción renal tubular y mayor susceptibilidad a las infecciones. Prácticamente cualquier glomerulopatía podría conducir a un síndrome nefrótico, pero es muy importante determinar si esta proteinuria es aislada o se asocia con la presencia de sedimentos (celulares o cilíndricos). Si hay presencia de un sedimento urinario persistente se debería tener en cuenta una glomerulonefritis primaria o secundaria a enfermedades sistémicas tipo lupus, o vasculitis. Si un paciente presenta proteinuria en el rango nefrótico y no se asocia con sedimentos anormales, siempre se debe tener en cuenta la posibilidad de una nefropatía diabética.

El lupus eritematoso sistémico ocasionalmente se asocia con glomerulonefritis membranosa y proteinuria sin sedimento. Cuando se han descartado otras posibilidades de glomerluopatías secundarias, como por ejemplo amiloidosis, nefrotoxicidad e inclusive hipertensión, se debe pensar en enfermedad glomerular primaria. La glomerulonefritis membranosa, la glomerulosclerosis focal segmentarla y la nefrosis lipoidea (o de mínimos cambios) son las causas primarias más comunes. La nefropatía por IgA también puede presentarse con proteinuria nefrótica, aunque la hematuria es lo más llamativo en este síndrome..$^{2021,27} \mathrm{El}$ diagnóstico de este tipo de proteinuria casi siempre requerirá una biopsia renal.

\section{Proteinuria como factor pronóstico}

Mucho se ha hablado del pronóstico clínico de la proteinuria $y$, actualmente, se considera que es el factor aislado más importante para determinar el avance y progresión de la enfermedad renal. La proteinuria es un factor de riesgo que acelera la insuficiencia renal. En el trasplante renal la proteinuria se relaciona con una menor supervivencia del injerto y del paciente. ${ }^{54}$ También se ha visto una correlación entre la tasa de proteinuria y la incidencia de muerte por factores cardiovasculares, de manera que una persona cardiópata con proteinuria tiene mayor riesgo de tener un síndrome coronario agudo, que una persona cardiópata sin proteinuria. En el INSIGHT (international nifedipine GITS study: intervention as a goal in hypertension treatment) se comparó el efecto sobre la morbimortalidad cardiovascular del tratamiento con nifedipina de liberación sostenida, con el del tratamiento diurético en 6.321 pacientes hipertensos de 55 a 80 años de edad, con al menos un factor de riesgo añadido. La proteinuria, definida por una excreción urinaria 0,5 gramos en 24 horas, ocasionó un pronóstico especialmente desfavorable al duplicar la probabilidad de sufrir un evento (riesgo relativo 2,08; IC 95\% 1,42-3,06). El riesgo añadido por la presencia de proteinuria fue superior al causado por otros factores de riesgo clásicos, como el consumo de tabaco, la diabetes o la hipertrofia ventricular izquierda e, incluso, superior al de situaciones de alto riesgo establecidas por el antecedente de enfermedad coronaria o de enfermedad arterial periférica. ${ }^{55,56}$ En el estudio LIFE (losartan intervention for endpoint reduction) se comparó la morbimortalidad cardiovascular $y$ total en pacientes hipertensos de 67 años de edad media con hipertrofia ventricular izquierda electrocardiográfica, según recibieran tratamiento basado en losartán o atenolol. ${ }^{57,58}$ En un análisis reciente del estudio se evaluó la relación entre la albuminuria, medida mediante el índice albúmina/creatinina, y el riesgo cardiovascular, y se observó un efecto deletéreo del nivel de excreción urinaria de albúmina desde los niveles más bajos, incluso en el rango de la normalidad..$^{59}$

En el estudio HOPE se determinó la excreción urinaria de albúmina mediante el índice albúmina/creatinina y se definió la microalbuminuria por un valor igual o superior a 2 $\mathrm{mg} / \mathrm{mmol}$. La prevalencia de microalbuminuria fue del $32,6 \%$ en los pacientes con diabetes y del $14,8 \%$ en los no diabéticos. La presencia de microalbuminuria ocasionó un riesgo aumentado de los eventos incluidos en la variable principal (muerte cardiovascular e infarto de miocardio o ictus no mortales), tanto en los diabéticos (riesgo relativo ajustado 1,97; IC del 95\% 1,68-2,31) como en los pacientes no diabéticos (riesgo relativo ajustado 1,61; IC del 95\% 1,36-1,90). La microalbuminuria confirió un riesgo relativo ajustado de muerte por cualquier causa de 2,09 (IC 95\% 1,84-2,38). También se describió un incremento progresivo del riesgo cardiovascular asociado a la microalbuminuria desde los niveles considerados normales, de tal forma que por cada aumento de $0,4 \mathrm{mg} / \mathrm{mmol}$ en el índice albúmina/ creatinina, se observó un aumento del 5,9\% en la probabilidad de sufrir un evento cardiovascular principal. Los autores del estudio concluyeron que cualquier grado de aumento en la albuminuria es un factor de riesgo cardiovascular en 
pacientes con enfermedad vascular establecida o con diabetes y otros factores de riesgo. ${ }^{60}$

Indistintintamente de la causa de la proteinuria, la esperanza de vida de una persona con proteinuria constante o progresiva es menor a la de la población general comparable. ${ }^{61}$

\section{Tratamiento}

Aún no existe un medicamento que actúe directamente sobre la proteinuria, pero el control de las patologías de fondo puede modificar el nivel de proteinuria. $^{53} \mathrm{Se}$ recomienda una dieta con entre 40 y $60 \mathrm{~g}$ de proteínas al día. Para los edemas se sugiere la restricción sódica y el uso de diuréticos. Se debe valorar el empleo de estatinas para la dislipidemias. $^{62}$ En pacientes con hipoalbuminemia importante es preciso considerar la profilaxis de anticoagulación: aspirina versus plavix. El buen manejo de la presión arterial es clave (PAS $110-130 \mathrm{mmHg}$ ) y para ello se utilizan principalmente los inhibidores de la enzima convertidora de la angiotensina, bloqueadores de los receptores de angiotensina II, y los beta-bloqueadores. ${ }^{63,64}$ En patologías autoinmunes y, sobre todo, en el caso de las glomerluopatias, se han utilizado diferentes tratamientos imunosupresores con respuestas variables.

\section{Abstract}

Proteinuria is defined as the urinary excretion of more than $150 \mathrm{mg}$ of protein in 24 hours. Proteinuria has been used as a marker of renal disease, becoming one of the nephrologists' most used markers. Common conditions such as arterial hypertension and Diabetes Mellitus express their renal damage as proteinuria, which has become a marker of systemic and not only renal disease. Normally, an individual filters $5000 \mathrm{mg}$ of proteins each day, of which $4950 \mathrm{mg}$ are reabsorbed in the proximal kidney tubule. In the present article we analyze the different types of proteinuria based on pathophysiological concepts. Currently, there are many laboratory methods for proteinuria quantification, but the proteinuria / creatinuria ratio and the 24 hour urine collection continue to be the more commonly utilized. Proteinuria has been shown to be individually the most important factor used to predict the progression of renal disease. The importance of proteinuria has also been confirmed in cardiac patients, becoming a risk factor for ischemic cardiovascular disease even more important than other classic ones such as smoking, diabetes or left ventricular hypertrophy. Proteinuria is more than just proteins in the urine, it's a sign of alert.

\section{Referencias}

1. Abuelo, J. Proteinuria: diagnostic principles and procedures. Ann Intern Med. 1983; 98: 186-91.

2. Miltenyi, M. Urinary protein excretion in healthy children. Clin Nephrol. 1979; 12: 216-21.

3. Bergstein, J. A practical approach to proteinuria. Pediatr Nephrol. 1999; 13: 697-700.

4. Clínica Universitaria de Navarra [home page on the Internet]. España. Análisis de las proteínas plasmáticas. Accedido 30 de abril de 2003. Disponible en: http://www.viatusalud.com/documento. asp? ID $=6229 \& \mathrm{G}=133$.

5. Giebisch, G. Windhager, E. Organization of the Urinary System. In: Boron, W. Boulpaep, E. editors. Medical Physiology. Elsevier Science; 2003. p $737-755$.

6. Horsey, P. The Cochrane 1998 albumin review - not all it was craked up to be. Euro J Anesth. 2002; 19: 170-704.

7. Evans, TW. Review article: albumin as a drug-biological effects of albumin unrelated to oncótica pressure. Aliment Phamacol Ther. 2002; 16:6-11.

8. Valtin, H. Schafer, J. Renal function. $3^{\text {rd }}$ ed. Boston: Little, Brown and Company. 1996.

9. Ulate, G. Fisiología renal. San José: Editorial de la Universidad de Costa Rica. 2006.

10. Tryggvason, K. Pettersson, E. Causes and consequences of proteinuria: the kidney filtration barrier and progressive renal failure. J Intern Med. 2003; 254:216-224.

11. Despopoulos, A. Silbernagl, S. Color Atlas of Physiology. $3^{\text {rd }}$ ed. Revised. New York: Thieme Inc. 1986.

12. Deen, W. Bridges, C. Brenner, B. Myers, B. Heteroporous model of glomerular size selectivity: application to normal and nephritic humans. Am J Physiol. 1985; 249: F374 - F389.

13. Ganong, W. Review of Medical Physiology. $22^{\text {ed }}$. New York: McGrawHill Medical. 2005.

14. Christensen, E. Birn, H. Megalin and cubulin: multifunctional endocitic receptors. Nature Review. 2002; 3: 258-267.

15. Devuyst O, Dahan K, Pirson Y. Tamm-Horsfall protein or uromodulin: new ideas about an old molecule. Nephrology dialysis transplantation. 2005 Jul; 20:1290-1294.

16. Kumar, S. Are Tamm-Horsfall protein and uromodulin identical. Euro J Clin Inv. 1998; 28: 483 - 484

17. Torffvit, O. Melander, O. Hulten, U. Urinary excretion rate of TammHorsfall protein is related to salt intake in humans. Nephron Physiol. 2004; 97: 31-36.

18. Zimmerbackl, L. Rostasy, K. Wiegele, G. Rasenack, A. Lohner, M. Brandis, M. Kinne, H. Tamm-Horsfall protein as a marker of tubular maturation. Pediatr Nephrol. 1996; 10: 448-452.

19. Wangsiripaisan, A. Role of polymeric Tamm-Horsfall protein in cast formation. Kidney Int. 2001; 59: 932 - 940.

20. Carroll, M. Temte, J. Proteinuria in Adults: A diagnostic approach. American Family Physician. 2000; 62: 1333-1341.

21. Venkat, K. Proteinuria and microalbuminuria in adults: Significance, evaluation and treatment. Southern Medical J. 2004; 97: 969 -978.

22. Yamasmit, W. Chaithongwongwatthana, S. Charoenvidhya, D. Uerpairojkit, B, Tolosa, J. Random urinary protein-to-creatinine ratio for prediction of significant proteinuria in women with Preeclampsia. J Maternal-Fetal and Neonatal Med. 2004; 16: 275-279.

23. Ginsberg JM, Chang BS, Matarese RA, Garella S. Use of single voided urine samples to estimate quantitative proteinuria. $\mathrm{N}$ EngI J Med 1983;309:1543-1546. 
24. Rodby RA, Rohde RD, Sharon Z, Pohl MA, Bain RP, Lewis EJ . The urine protein to creatinine ratio as a predictor of 24-hour urine protein excretion in type 1 diabetic patients with nephropathy. The Collaborative Study Group. Am J Kidney Dis 1995;26:904-909.

25. Somanathan, N. Farrel, T. Galimerti, A. A comparison between 24hour and 2-hour urine collection for the determination of proteinuria. J Obs and Gyn. 2003; 23: 378-380.

26. Breckin, C. Garber, S. Primer of Urinalysis: III. Proteinuria - promises and pitfallas. Kidney. 2002 Update I: 95- 97.

27. D’Amico, G. Bazzi, C. Pathophysiology of proteinuria. Kidney Int. 2003; 63: 809 - 825.

28. Marks, M. Mclaine, P. Drummond, K. Proteinuria in children with febrile illnesses. Arch Dis Child. 1970; 45:250-253.

29. Coye, R. Rosandich, R. Proteinuria during the 24-hour period following exercise. J Appl Physiol. 1960; 15:592-594.

30. Poortmans, J. Postexcersie proteinuria in humans. JAMA. 1985; 253: 236-240.

31. Lindheimer, M. Katz, A. The kidney and hypertension in pregnancy. In: Brenner, B. Rector, F. editors. The kidney. $4^{\text {th }}$ ed. Philadelphia: WB Saunders C.; 1991. p.1551- 1595.

32. Gordon, M. Maternal physiology in pregnancy. In: Gabbe, S. Obstetrics: Normal and Problem Pregnancies. $4^{\text {th }}$ ed. Philadelphia: Churchill Livingstone; 2002: p.63 - 92.

33. Reuben, D. Watchel, T. Brown, P. Transient proteinuria inemergency medical admissions. N Engl J Med. 1982; 306: 1031-1033.

34. Randolph, M. Greenfield, M. Proteinuria: a six year study of normal infants, preschool and school age populations previously screened for urinary tract disease. Am J Dis Child. 1967; 114:631-638. Abstract.

35. Von Bonsdorff M, Koskenvuo K, Salmi HA, Pasternack A. Prevalence and causes of proteinuria in 20-year old Finnish men. Scand J Urol Nephrol 1981;15:285-90.

36. Levitt JI. The prognostic significance of proteinuria in young college students. Ann Intern Med 1967;66:685-96. Abstract.

37. Muth RG. Asymptomatic mild intermittent proteinuria. Arch Intern Med 1965;115:569-74.Abstract.

38. Rytand DA, Speiter S. Prognosis in postural (orthostatic) proteinuria. N Engl J Med 1981;305:618-21.

39. Springberg PD, Garrett LE, Thompson AL, Collins NF, Lordon RE, Robinson RR . Fixed and reproducible proteinuria: results of a 20year follow-up study. Ann Intern Med 1982;97:516-9.

40. Vehaskari VM, Rapola J. Isolated proteinuria: Analysis of a schoolage population. J Pediatr 1982;101:661-8.

41. Houser MT, Jahn MF, Kobayashi A, Walburn J. Assessment of urinary protein excretion in the adolescent: Effect of body position and exercise. J Pediatr 1986;109:556-61.

42. Vehaskari VM. Mechanism of orthostatic proteinuria. Pediatr Nephrol 1990;4:328-30.

43. Devarajan, P. Mechanisms of orthostatic proteinuria: lessons from a trasplant donor. J Am Soc Nephrol. 1993; 4:36-39.

44. Sinniah R, Law CH, Pwee HS. Glomerular lesions in patients with asymptomatic persistent and orthostatic proteinuria discovered on routine medical examinations. Clin Nephrol 1977;7:1-14.

45. Vehaskari V. Mechanism of orthostatic proteinuria. Pediatr Nephrol 1990; 4: 328-330.

46. Shintaku N, Takahashi Y, Akaishi K, Sano A, Kuroda Y. Entrapment of left renal vein in children with orthostatic proteinuria. Pediatr Nephrol 1990; 4: 324-327.

47. Trachtman H, Bergwerk A, Gauthier B. Isolated proteinuria in children. Clin Pediatr 1994;33:468-72.

48. Urizar RE, Tinglof BO, Smith Jr FG, McIntosh RM. Persistent asymptomatic proteinuria in children. Am J Clin Pathol 1974;62:46171.
49. Yoshikawa N, Kitagawa $\mathrm{K}$, Ohta $\mathrm{K}$, Tanaka $\mathrm{R}$, Nakamura $\mathrm{H}$ Asymptomatic constant isolated proteinuria in children. J Pediatr 1991;119:375-9.

50. Yoshikawa N, Uehara S, Yamana K, Ikeuchi H, Hiraumi M, Matsuo T, et al. Clinicopathological correlations of persistent asymptomatic proteinuria in children. Nephron 1980;25:127-33.

51. Kusumoto Y, Takebayashi S. Benign persistent asymptomatic proteinuria with incomplete foot process disease in adolescents: a new clinical entity? Clin Nephrol 1987;28:174-9.

52. Mogensen CE. Microalbuminuria predicts clinical proteinuria and early mortality in maturity-onset diabetes. $\mathrm{N}$ Engl $\mathrm{J}$ Med 1984;310:356.

53. Krasper, D. Braunwald, E. Fauci, A. Hauser, S. Longo, D. Jameson, L. Principles of Internal Medicine. 16 ${ }^{\text {th }}$ ed. 2004. McGraw-Hill Professional

54. Universidad Complutense de Madrid. [home page on the Internet]. España. Proteinuria y transplante renal. 2002. Disponible en: http:// www.ucm.es/info/fmed/medicina.edu/Temasinteres/proteina.htm

55. Brown MJ, Palmer CR, Castaigne A, de Leeuw PW, Mancia G, Rosenthal T, et al. Morbidity and mortality in patients randomised to double-blind treatment with a long-acting calcium-channel blocker or diuretic in the International Nifedipine GITS study: Intervention as a Goal in Hypertension Treatment (INSIGHT). Lancet 356: 366-372, 2000 .

56. Segura J, Campo C, Ruilope LM: Proteinuria: an underappreciated risk factor in cardiovascular disease. Curr Cardiol Rep 4: 458-462, 2002.

57. Dahlöf B, Devereux RB, Kjeldsen SE, Julius S, Beevers G, de Faire $\mathrm{U}$, et al. Cardiovascular morbidity and mortality in the Losartan Intervention For Endpoint reduction in hypertension study (LIFE): a randomized trial against atenolol.. Lancet. 2002 Mar23;359(9311):9951003

58. Lindholm LH, Ibsen H, Dahlöf B, Devereux RB, Beevers G, de Faire $\mathrm{U}$, et al. Cardiovascular morbidity and mortality in patients with diabetes in the Losartan Intervention For Endpoint reduction in hypertension study (LIFE): a randomised trial against atenolol. Lancet. 2002 Mar 23;359(9311):1004-10

59. Wachtell K, Ibsen H, Olsen MH, Borch-Johnsen K, Lindholm LH, Mogensen CE, et al. Albuminuria and cardiovascular risk in hypertensive patients with left ventricular hypertrophy: the LIFE study. Ann Intern Med. 2003 Dec 2;139:901-6.

60. Gerstein HC, Mann JFF, Yi Q, Zinman B, Dinneen SF, Hoogwerf B, et al. Albuminuria and risk of cardiovascular events, death, and heart failure in diabetic and nondiabetic individuals. JAMA $2001 \mathrm{Jul}$ 25;286:421-6.

61. Iseki, K. Proteinuria and the risk of developing end stage renal disease. Kidney Int 2003; 63: 1468-1474.

62. Hart, P. Maximum reduction of proteinuria in renal disease. Kidney 2004; 13: $99-103$.

63. Mulrow, C. Guiding lights for antihypertensive treatment in patients with nondiabetic chronic renal disease. Ann Int Med. 2003; 139: 296 $-298$.

64. Hogg, R. Portman, R. Milliner, D. Lemley, K. Eddy, A. Ingelfinger, J. Evaluation and management of pronteinuria and nephritic syndrome in children: recomendationes from a pediatric nephrology panel established at the national kidney foundation conference on proteinuria, albuminuria, risk, assesmente, detection and elimination (PARADE). Pediatrics. 2000; 105:1242-1249. 